\title{
PDES IN THE INVERSE PROBLEM OF DYNAMICS
}

\author{
Mira-Cristiana Anisiu \\ T. Popoviciu Institute of Numerical Analysis \\ Romanian Academy \\ 37 Republicii st., Cluj-Napoca, RO-3400 Romania \\ mira@math.ubbcluj.ro
}

\begin{abstract}
The basic equations are exposed for the following version of the inverse problem of dynamics: determine the two-dimensional potential compatible with a given family of orbits, traced by a material point. If the potential is known in advance, a nonlinear equation is satisfied by the function representing the family of orbits. Its solutions are studied in the presence of additional information on the family. The possibility of programming the motion of a material point in a preassigned region of the plane is also considered.
\end{abstract}

Keywords: inverse problem of dynamics, Szebehely's and Bozis' equations.

\section{Introduction}

The version of the inverse problem of dynamics discussed in this paper consists in finding the two-dimensional potential which governs the motion of a dynamical system, knowing a given family of orbits. The first outstanding results are due to Newton (1687), who found the force law compatible with Kepler's laws. The paper which gave a new impulse to the field of the inverse problems was that of Szebehely's (1974). Information on various other aspects (nonconservative systems, threedimensional ones, rotating frames, holonomic systems with $n$ degrees of freedom etc) of the inverse problem is contained in Bozis (1995) and Anisiu (1998).

\section{The main tools of the inverse problem}

Given a family of curves

$$
f(x, y)=c
$$

The original version of this chapter was revised: The copyright line was incorrect. This has been corrected. The Erratum to this chapter is available at DOI: 10.1007/978-0-387-35690-7_44

V. Barbu et al. (eds.), Analysis and Optimization of Differential Systems

(C) IFIP International Federation for Information Processing 2003 
one looks for a potential for which this family is an orbit family of a particle. The problem is then to determine the potential $V \in C^{1}(D)$ for which the equations of the motion of a unit mass particle are

$$
\ddot{x}=-V_{x} \quad \ddot{y}=-V_{y},
$$

knowing a given family of orbits (2.1). The first order partial differential equation satisfied by the potential $V$ was obtained by Szebehely (1974).

The system (2.2) admits the energy integral $\dot{x}^{2}+\dot{y}^{2}=2(E(f)-V)$, $E(f)$ being constant on every orbit in (2.1).

Theorem 2.1 Let $D \subset \mathbb{R}^{2}$ be an open set and $f \in C^{2}(D)$ such that $f_{x}^{2}(x, y)+f_{y}^{2}(x, y) \neq 0$ for each $(x, y) \in D$. If the system (2.2) admits as orbits the curves of the family (2.1), then the function $V$ satisfies the partial differential equation

$$
f_{x} V_{x}+f_{y} V_{y}-\frac{2(E(f)-V)}{f_{x}^{2}+f_{y}^{2}}\left(f_{x x} f_{y}^{2}-2 f_{x y} f_{x} f_{y}+f_{y y} f_{x}^{2}\right)=0 .
$$

Proof. From (2.1) we obtain $\dot{x} f_{x}+\dot{y} f_{y}=0$, hence $\dot{x}$ and $\dot{y}$ will be

$$
\dot{x}= \pm \sqrt{k} f_{y} \quad \dot{y}=\mp \sqrt{k} f_{x},
$$

where the sign depends on the sense of the motion on the orbit, and $k \in C^{1}\left(D, \mathbb{R}_{+}\right)$is an arbitrary function. Differentiating again we have

$$
\begin{aligned}
& \ddot{x}=k\left(f_{x y} f_{y}-f_{y y} f_{x}\right)+\left(k_{x} f_{y}-k_{y} f_{x}\right) f_{y} / 2 \\
& \ddot{y}=k\left(f_{x y} f_{x}-f_{x x} f_{y}\right)-\left(k_{x} f_{y}-k_{y} f_{x}\right) f_{x} / 2 .
\end{aligned}
$$

It follows

$$
\begin{aligned}
& -V_{x}=k\left(f_{x y} f_{y}-f_{y y} f_{x}\right)+\left(k_{x} f_{y}-k_{y} f_{x}\right) f_{y} / 2 \\
& -V_{y}=k\left(f_{x y} f_{x}-f_{x x} f_{y}\right)-\left(k_{x} f_{y}-k_{y} f_{x}\right) f_{x} / 2
\end{aligned}
$$

and

$$
f_{x} V_{x}+f_{y} V_{y}=-k\left(2 f_{x y} f_{x} f_{y}-f_{x}^{2} f_{y y}-f_{y}^{2} f_{x x}\right) .
$$

From the energy integral and the relations (2.4), the function $k$ is given by $k=2(V-E(f)) /\left(f_{x}^{2}+f_{y}^{2}\right)$. We replace it in (2.5) and obtain Szebehely's equation (2.3).

Remark 2.1 In the paper Bozis (1983) the functions

$$
\gamma=\frac{f_{y}}{f_{x}} \quad \Gamma=\gamma \gamma_{x}-\gamma_{y}
$$

were introduced for the family of curves (2.1). Using these notations, Szebehely's equation was written in the simpler form (Bozis, 1983)

$$
V_{x}+\gamma V_{y}+\frac{2 \Gamma(E-V)}{1+\gamma^{2}}=0 .
$$


From the equation above, an inequality can be deduced because the kinetic energy of the particle $B=E-V$ is nonnegative.

Corollary 2.1 The potential $V$ satisfies the inequality (Bozis and Ichtiaroglou, 1994)

$$
\Gamma\left(V_{x}+\gamma V_{y}\right) \leq 0
$$

In what follows the functions are considered to be sufficiently smooth. The equation (2.7) has the disadvantage that it contains the energy $E$, which in general is not known in advance. Bozis (1984) has eliminated the energy, obtaining a second order partial differential equation.

Theorem 2.2 Denoting $\kappa=\frac{1}{\gamma}-\gamma, \quad \lambda=\frac{\Gamma_{y}-\gamma \Gamma_{x}}{\gamma \Gamma}, \quad \mu=\lambda \gamma+\frac{3 \Gamma}{\gamma}$, the potential $V$ satisfies the second order equation (Bozis, 1984)

$$
-V_{x x}+\kappa V_{x y}+V_{y y}=\lambda V_{x}+\mu V_{y} \text {. }
$$

Proof. The energy $E=E(f)$ can be eliminated from equation (2.7) by solving it with respect to $E$ and inserting $E$ into

$$
E_{y}=\gamma E_{x}
$$

the slope function $\gamma$ being given by (2.6). Relation (2.10) holds because from $E=E(f)$ we have $E_{x}=E^{\prime} f_{x}$ and $E_{y}=E^{\prime} f_{y}$.

The main tools used in the inverse problem are: Szebehely's quasilinear first order partial differential equation (2.7), Bozis' linear second order partial differential equation (2.9), and the inequality (2.8). If one manages to obtain a solution of (2.9), from (2.7) one can find the value of the energy, and from (2.8) the plane region where the actual trajectories are allowed to take place.

Example 2.1 For the case of the family $f(x, y)=x^{2}+y^{2}$ of concentric circles, Broucke and Lass (1977) found (in polar coordinates $r, \theta$ ) the potential $V(r, \theta)=g(r)+h(\theta) / r^{2}$, where $g$ and $h$ are arbitrary functions. The energy function is in this case $E=g(r)+r g^{\prime}(r) / 2$, and the allowed region obtained from (2.8) is given by $g^{\prime}(r)-2 h(\theta) / r^{3} \geq 0$. Other cases in which Szebehely's equation is solvable are exposed in Grigoriadou et al (1999).

The relation (2.9) was derived only for the case $\Gamma \neq 0$. From (2.6) one can express $\Gamma$ in terms of the derivatives of $f$ as

$$
\Gamma=\frac{2 f_{x y} f_{x} f_{y}-f_{x x} f_{y}^{2}-f_{y y} f_{x}^{2}}{f_{x}^{3}},
$$


hence the family (2.1) consists of straight lines if and only if $\Gamma=0$; in view of (2.6) this condition may be written as

$$
\gamma \gamma_{x}-\gamma_{y}=0 \text {. }
$$

\section{Families of straight lines}

The problem of determining the potentials under whose action families of straight lines are described was considered by Bozis and Anisiu (2001).

Szebehely's equation (2.7) for a family of straight lines becomes

$$
V_{x}+\gamma V_{y}=0 \text {. }
$$

Remark 3.1 In what follows we shall consider $\gamma \neq 0$, because for $\gamma=0$ we obtain $V_{x}=0$, hence $V=v(y)$; this potential produces only the family of vertical straight lines $x=c$.

We differentiate (3.12) with respect to $x$, then to $y$, and express $\gamma_{x}$ and $\gamma_{y}$ from the obtained equations. From (2.11) we get

$$
-V_{x x}+\left(\frac{1}{\gamma}-\gamma\right) V_{x y}+V_{y y}=0 ;
$$

this corresponds to Bozis' partial differential equation for the straight lines situation. We insert $\gamma$ obtained from (3.12) into (3.13) and get a nonlinear partial differential equation

$$
V_{x} V_{y}\left(V_{x x}-V_{y y}\right)=V_{x y}\left(V_{x}^{2}-V_{y}^{2}\right)
$$

which must be satisfied by all potentials creating (among other orbits) a family of straight lines.

Remark 3.2 Given an adequate $\gamma$ (i.e. satisfying (2.11)), there exist infinitely many potentials $V(x, y)$, found from (3.12), creating the monoparametric family of straight lines. But for an adequate $V$ (i.e. satisfying (3.14) and depending on both variables $x$ and $y$ ), there corresponds to it exactly one $\gamma$, found from (3.12), hence one family of straight lines.

Focussing our attention on equation (3.14), we mention some of its obvious solutions:

(i) $V=V(x)$ or $V=V(y)$;

(ii) $V=V\left(k_{1} x+k_{2} y\right), k_{1}$ and $k_{2}$ constants;

(iii) $V=x^{2}+y^{2}$.

In case (i), equation (3.12) can be satisfied only for the trivial case, $V=$ const, as follows from Remark 3.1. For the class of potentials in case (ii), we obtain from (3.12) the family of straight lines given 
by $\gamma=-\frac{k_{1}}{k_{2}}=$ const. In case (iii), the family of straight lines has a homogeneous of order 0 slope function $\gamma=-\frac{x}{y}$. The special solution $V=x^{2}+y^{2}$ allows us to state that all central potentials $V=V(r)$ are solutions of (3.14).

Other classes of potentials, some expressed in polar coordinates, are given in Bozis and Anisiu (2001).

\section{Equations of the direct problem}

Bozis' equation represents a relation between the function $\gamma$ and the potential $V$. It can be used to face the direct problem of Dynamics: given a potential $V$, find the families of orbits which can be generated. The nonlinear second order differential equation relating potentials and orbits in the form suitable for the direct problem (Bozis, 1995) is

$$
\begin{aligned}
& \gamma^{2} \gamma_{x x}-2 \gamma \gamma_{x y}+\gamma_{y y}=\frac{\gamma \gamma_{x}-\gamma_{y}}{V_{y} \gamma+V_{x}} \\
& \left(-\gamma_{x} V_{x}+\left(2 \gamma \gamma_{x}-3 \gamma_{y}\right) V_{y}+\gamma\left(V_{x x}-V_{y y}\right)+\left(\gamma^{2}-1\right) V_{x y}\right)
\end{aligned}
$$

This is obtained by rearranging Bozis' equation (2.9).

Due to its nonlinearity in $\gamma$, it is difficult to be solved. Additional information may help in searching for solutions. The case of families produced by homogeneous potentials was considered by Bozis and Stefiades (1993), and by Bozis and Grigoriadou (1993); the problem was reduced to solving ordinary differential equations. Homogeneous families produced by inhomogeneous potentials were studied by Bozis et al (1997), as well as families of orbits with $\gamma=\gamma(x)$, corresponding to families $f(x, y)=y+h(x)=c$ (Bozis et al, 2000); in these two cases $\gamma$ was found as the common root of some algebraic equations in $\gamma$, with coefficients depending on $V$ and on its derivatives.

The additional condition satisfied by $\gamma$ may be put in the terms of a first order differential equation. Indeed, if $f$ is homogeneous of degree $m$, then $\gamma$ is homogeneous of degree 0 . This happens if and only if $x \gamma_{x}+y \gamma_{y}=0$. For the family $f(x, y)=y+h(x)$ the corresponding $\gamma$ is given by $\gamma=\frac{1}{h^{\prime}(x)}$ and satisfies the equation $\gamma_{y}=0$.

More generally, we can suppose that we have additional information on the family of curves (2.1) given as a linear first order differential equation which is satisfied by $\gamma, a(x, y) \gamma_{x}+b(x, y) \gamma_{y}=0$. In this case, as in the special cases mentioned above, if the potential satisfies a differential condition, the family $\gamma$ can be obtained as a common solution of two polynomial equations of degree at most seven, respectively twelve (Bozis et al, 2002). The coefficients of the polynomials in $\gamma$ are expressions containing the derivatives of $V$ up to the forth order, and can be calculated using symbolic algebra programs. 
Example 4.1 For the Hénon-Heiles potential $V(x, y)=\frac{1}{2} x^{2}+8 y^{2}+$ $x^{2} y+\frac{16}{3} y^{3}$ and $a(x, y)=x, b(x, y)=y$, the solution $\gamma=-\frac{x}{4 y}$ corresponding to the family $y x^{-4}=c$ was found by Bozis and al (1997) as an example of a homogeneous family traced under the action of an inhomogeneous potential. The energy on the family is given by $E=-\frac{1}{24 c}$ and the allowed region is $\left(x^{2}+8 y^{2}+12 y\right) y \leq 0$.

\section{Programmed motion}

It was proved in section 2 that during the motion of a material point of unit mass along an orbit of the family (2.1), the inequality

$$
B(x, y) \geq 0
$$

must be observed, with

$$
B=E(f(x, y))-V(x, y) .
$$

This means that the motion is allowed along those members of the family (2.1) which are lying only inside some regions of the $x y$ plane. The function $B(x, y)$ is the kinetic energy of the material point of unit mass, as it moves on any of the orbits in the presence of the potential $V(x, y)$.

According to Galliulin (1984), dynamical systems with programmed motion "are solved in such a way that the process occurring in these systems satisfies some preset requirements". The requirement that the motion takes place in the region (5.16) was considered at first by Bozis (1996). This type of programmed motion is the following: Given a preassigned region in the plane defined by (5.16), find a potential $V$ which produces as trajectories of (2.2) the curves in the family (2.1).

Anisiu and Bozis (2000) studied this problem completely for a simpler family of functions, given by

$$
f(x, y)=y-h(x)=c
$$

where $h$ is a nonlinear $\left(h^{\prime \prime}(x) \neq 0\right)$ function of $x$. A simpler function $b$, which is nonnegative if and only if $B$ is nonnegative, was considered. Under certain conditions on $b$, the function $h$ (and, consequently, the family $f$ ) as well as the energy dependence function $E(f)$ and the potential $V(x, y)$ were determined. Let us denote

$$
b=B /\left(1+\gamma^{2}\right) \text {. }
$$

The function $b$ satisfies a second order partial differential equation for the general family of functions (2.1). This was derived at first by Bozis (1995) starting from the case of nonconservative forces. Here we shall obtain it using Szebehely's equation.

Theorem 5.1 Denoting $\kappa=\frac{1}{\gamma}-\gamma, \quad m=2 \gamma_{y}+\frac{\gamma_{x}}{\gamma}, n=2 \gamma_{x}-\frac{3 \gamma_{y}}{\gamma}$, $p=\frac{2 \Gamma_{y}}{\gamma}$, the function $b$ satisfies the linear second order partial differential equation (Bozis, 1995)

$$
-b_{x x}+\kappa b_{x y}+b_{y y}=m b_{x}+n b_{y}+p b .
$$


Proof. In view of (5.17) and (5.19), Szebehely's equation (2.7) becomes

$$
V_{x}+\gamma V_{y}=-2 \Gamma b
$$

and $E=V+\left(1+\gamma^{2}\right) b$. But $\gamma=\frac{E_{y}}{E_{x}}$, so we obtain $\gamma=\frac{V_{y}+\left(\left(1+\gamma^{2}\right) b\right)_{y}}{V_{x}+\left(\left(1+\gamma^{2}\right) b\right)_{x}}$, which can be written as

$$
\gamma V_{x}-V_{y}=\left(1+\gamma^{2}\right)\left(b_{y}-\gamma b_{x}\right)-2 \gamma \Gamma b .
$$

Solving the system of two equations (5.21) and (5.22) we get $V_{x}=$ $\gamma\left(b_{y}-\gamma b_{x}\right)-2 \Gamma b, \quad V_{y}=\gamma b_{x}-b_{y}$. Writing the compatibility condition $\left(V_{x}\right)_{y}=\left(V_{y}\right)_{x}$ we obtain $(5.20)$.

We can rearrange (5.20) as a nonlinear equation of order two in $\gamma$

$$
\begin{gathered}
2 b \gamma \gamma_{x y}-2 b \gamma_{y y}=-2 b \gamma_{x} \gamma_{y}-\left(2 b_{y} \gamma+b_{x}\right) \gamma_{x} \\
-\left(2 b_{x} \gamma-3 b_{y}\right) \gamma_{y}-b_{x y} \gamma^{2}+\left(b_{y y}-b_{x x}\right) \gamma+b_{x y}
\end{gathered}
$$

which can be used in the case when the function $b$ is known.

Considering the kinetic energy $B=1$ (or, equivalently, $B=$ const) for all the curves of the family (2.1), equation (5.20) will become

$$
\left(1+\gamma^{2}\right)\left(\gamma^{2} \gamma_{x x}-2 \gamma \gamma_{x y}+\gamma_{y y}\right)+2 \gamma\left(1-\gamma^{2}\right) \gamma_{x}^{2}-4 \gamma \gamma_{y}^{2}+2\left(3 \gamma^{2}-1\right) \gamma_{x} \gamma_{y}=0
$$

which is the equation giving the totality of isotach orbits (Bozis, 1986).

For the special case of the family (5.18), the function $z$ in Anisiu and Bozis (2000) is equal to $-\gamma$, and equation (5.20) becomes

$$
\left(b_{x}-2 b_{y} z\right) z^{\prime}=b_{x y} z^{2}+\left(b_{y y}-b_{x x}\right) z-b_{x y},
$$

which was derived there directly. The method presented in the cited paper gives the family of orbits, the energy and the potential, when a suitable function $b$ defines the allowed region.

Example 5.1 For $x>0$ and the function $b=b_{2} y^{2}+b_{1} y+b_{0}$, with $b_{2}=-3 / x, b_{1}=3\left(x^{4}+1\right) / x^{2}, \quad b_{0}=-\left(3 x^{4}\left(x^{4} / 5+1\right)+1\right) / x^{3}$, the family of orbits of the form (5.18) $y-\frac{1}{x}=c$ is described inside the region $b(x, y) \geq 0$. In this case, $E=c^{3}$ and the potential is $V(x, y)=$ $y^{3}+3 x^{3} y^{2}-3 x^{2}\left(x^{4}+2\right) y+\frac{1}{5} x\left(3 x^{8}+18 x^{4}+20\right)$.

\section{Final remarks}

PDEs appear in connection with the inverse problem of dynamics; (2.7) is quasilinear and (2.9) is linear in the potential function $V$. The direct problem gives rise to the nonlinear equation (4.15). Other PDEs are produced by related problems: the study of potentials creating families of straight lines leads to equation (3.14), the programming of the 
motion in certain regions of the plane to (5.20), (5.23), and the study of families traced with constant kinetic energy to (5.24).

Acknowledgement. This research was partially supported by the Ministry of Education and Research, by grant 343-CNCSIS 33444/2002.

\section{References}

[1] Anisiu, M.-C. : 1998, Nonlinear Analysis Methods with Application in Celestial Mechanics, University Press, Cluj-Napoca (in Romanian)

[2] Anisiu, M.-C. and Bozis, G. : 2000, Programmed motion for a class of families of planar orbits, Inverse Problems 16, 19-32

[3] Bozis, G. : 1983, Inverse problem with two-parametric families of planar orbits, Celest. Mech. 31, 129-143

[4] Bozis, G. : 1984, Szebehely's inverse problem for finite symmetrical material concentrations, Astronom. Astrophys. 134, 360-364

[5] Bozis, G. : 1986, Adelphic potentials, Astron. Astrophys. 160, 107-110

[6] Bozis, G. and Grigoriadou, S. : 1993, Families of planar orbits generated by homogeneous potentials, Celest. Mech. 57(3), 461-472

[7] Bozis, G. and Stefiades, Ap.: 1993, Geometrically similar orbits in homogeneous potentials, Inverse Problems 9(2), 233-240

[8] Bozis, G. and Ichtiaroglou, S.: 1994, Boundary curves for families of planar orbits, Celest. Mech. 58, 371-385

[9] Bozis, G. : 1995, The inverse problem of dynamics: basic facts, Inverse Problems 11, 687-708

[10] Bozis, G.: 1996, Two-dimensional programmed motion, Proceedings of the 2nd Hellenic Astronomical Conference, Thessaloniki, June 29-July 1, 1995 (eds. M. E. Contadakis et al), pp. 587-590

[11] Bozis, G., Anisiu, M.-C. and Blaga, C. : 1997, Inhomogeneous potentials producing homogeneous orbits, Astron. Nachr. 318, 313-318

[12] Bozis, G., Anisiu, M.-C. and Blaga, C. : 2000, A solvable version of the direct problem of dynamics, Rom. Astronom. J. 10(1), 59-70

[13] Bozis, G. and Anisiu, M.-C. : 2001, Families of straight lines in planar potentials, Rom. Astronom. J. 11(1), 27-43

[14] Bozis, G., Anisiu, M.-C. and Blaga, C. : 2002, Special families of orbits in the direct problem of dynamics, preprint

[15] Broucke, R. and Lass, H. : 1977, On Szebehely's equation for the potential of a prescribed family of orbits, Celest. Mech. 16, 215-225

[16] Galiullin, A. S. : 1984, Inverse Problem of Dynamics, p. 91, Mir Publishers, Moscow

[17] Grigoriadou, S., Bozis, G. and Elmabsout, B.: 1999, Solvable cases of Szebehely's equation, Celest. Mech. 74, 211-221

[18] Newton, I. : 1687, Philosophiae Naturalis Principia Mathematica, London

[19] Szebehely, V. : 1974, On the determination of the potential by satellite observations, in E. Proverbio (ed.) Proc. Intern. Meeting on Earth's Rotations by Satellite Observations, The University of Cagliari, Bologna, Italy, 31-35. 